\title{
EXPERIMENTAL WORK AT THE UNIVERSITY OF GHIGAGO ON THE ONSET OF THERMAL INSTABILITY IN A LAYER OF FLUID HEATED FROM BELOW
}

\author{
YOSHINARI NAKAGAWA \\ Enrico Fermi Institute of Nuclear Studies, University of Chicago, U.S.A. \\ AND \\ KEVIN H. PRENDERGAST \\ Yerkes Observatory, University of Chicago, U.S.A.
}

This paper will summarize the experimental work at the University of Chicago on the problem of the onset of thermal instability in a layer of fluid heated from below. The purpose of this work has been to test certain theoretical predictions of the Rayleigh number at which instability sets in, and to determine the type of instability which appears at the critical point. The earlier experiments of this series were done at the hydrodynamics laboratory of the University of Chicago in connexion with a program of meteorological reseach $[1,2,3,4]$. The current work is being done at the newly organized hydromagnetics laboratory of the Enrico Fermi Institute of Nuclear Studies. This laboratory utilizes the magnet of the old Chicago cyclotron, with pole pieces $92.7 \mathrm{~cm}$ in diameter and a gap of $22.1 \mathrm{~cm}$. The magnet was reconstructed to allow the field strength to be varied from $o$ to 13,000 gauss; the field is uniform to better than $\mathrm{I} \%$ over the experimental area. The new laboratory is under the administrative supervision of Professors S. K. Allison and S. Chandrasekhar; the experiments are being done by $\mathrm{Y}$. Nakagawa. The theoretical investigations are primarily the work of Chandrasekhar $[5,6,7,8,9,10]$ and it will be convenient to review some of his results before discussing the experiments.

The general situation which is envisaged is the following: suppose that a horizontal layer of fluid is heated from below in such a way as to establish a mean adverse temperature gradient $\beta=-d T / d z$. In addition to the force of gravity $\mathbf{g}$ acting on the fluid there may be a prevailing magnetic field $\mathbf{H}$, or the layer may be in rotation about some axis with an angular velocity $\boldsymbol{\Omega}$. The fluid may be confined between rigid boundaries, 
and off are indicated. In each case the value of $\beta$ is estimated from the highest portion of the trace. Plate II is taken from the experimental data on overstability in a rotating layer of mercury, [2] again with $\beta<\beta_{c}$ (top), $\beta \simeq \beta_{c}$ and $\beta>\beta_{c}$. Notice particularly the wiggles which appear in the middle and bottom traces - these establish the presence of overstability and enable the period of oscillation to be determined. An enlargement of the last trace appears in Plate III.

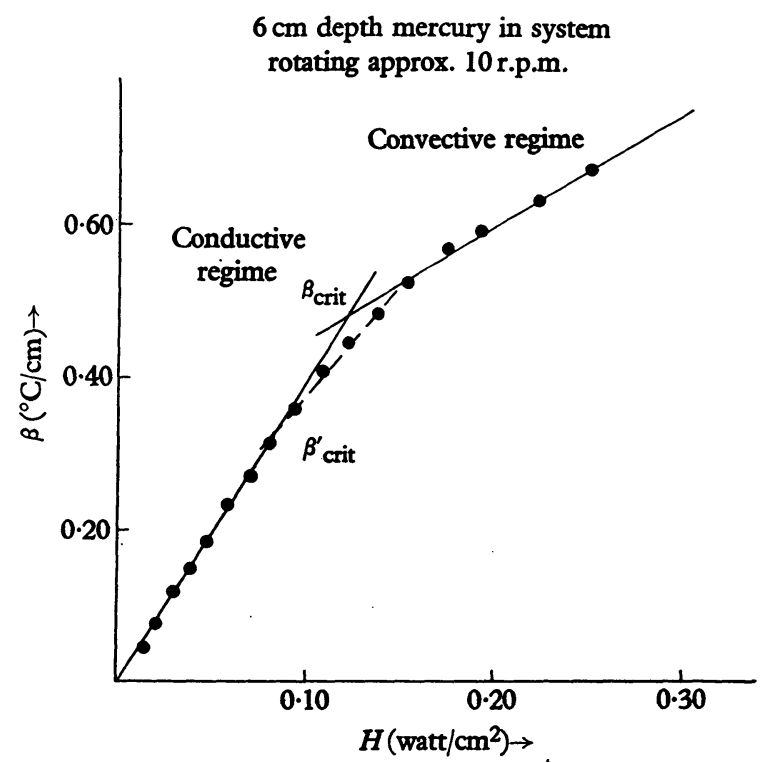

Fig. 1. Determination of $\beta_{c}$. (From D. Fultz and Y. Nakagawa [2].)

The final determination of $\beta_{c}$ is facilitated by plotting $\beta$ against the heating rate, as shown in Fig. I. For the case illustrated, $\beta$ is a linear function of the heating rate $H$ for $H<0.08$ watts $/ \mathrm{cm}^{2}$ (corresponding to a definitely conductive regime), and again for $H>0 \cdot 16$ watts $/ \mathrm{cm}^{2}$ (corresponding to convection). Between these limits, however, there is a transition region in which it is possible that the convective cells have started to form near the bottom of the fluid but do not yet extend to the top. The adopted value of $\beta_{c}$ is obtained by extrapolating the linear portions of the curve.

The procedure outlined above is sufficient to determine one value of the critical Rayleigh number. The experiment on thermal instability in a rotating liquid involved the determination of $R_{c}$ as a function of the Taylor number for both the convective and overstable cases. The results are presented in Figs. 2 and 3, and it may be seen that the theoretical 


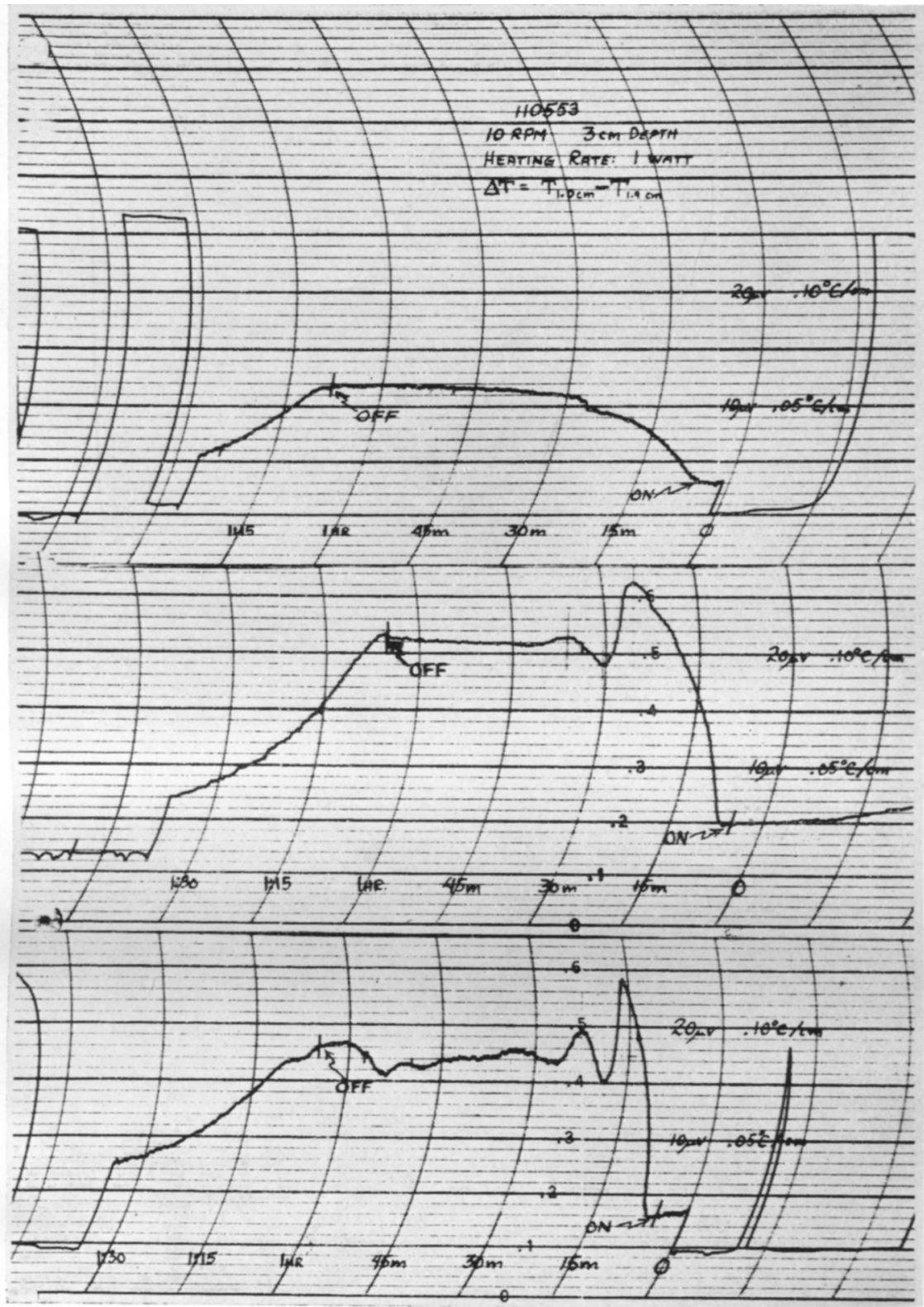

Plate I. Convection in a rotating layer of water: adverse temperature gradient $\beta$ as a function of time for $\beta<\beta_{c}$ (top), $\beta \simeq \beta_{c}$ (middle) and $\beta>\beta_{c}$ (bottom). The time increases to the left. 


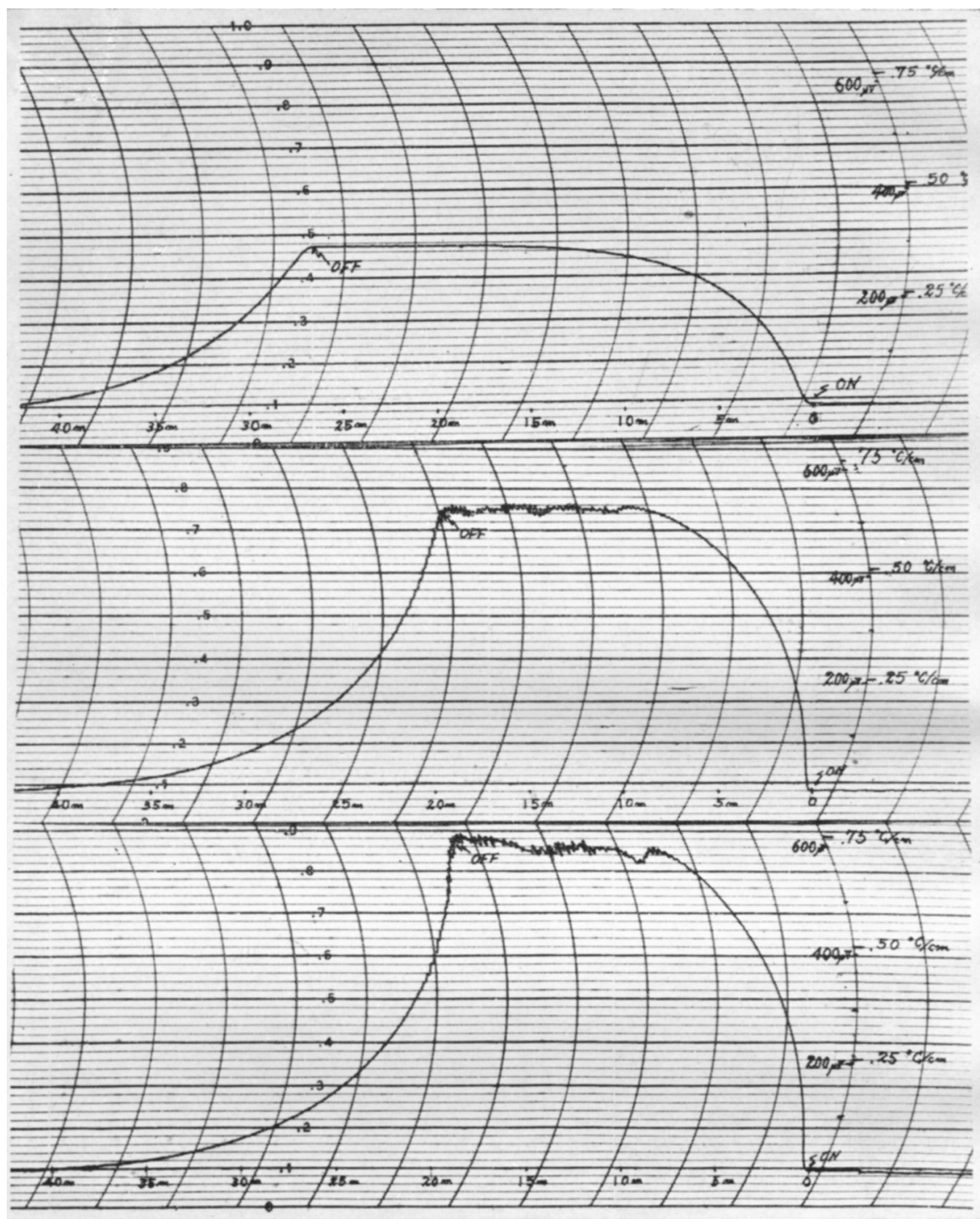

Plate II. Overstability in a rotating layer of mercury: adverse temperature gradient $\beta$ as a function of time for $\beta<\beta_{c}$ (top), $\beta \simeq \beta_{c}$ (middle) and $\beta>\beta_{c}$ (bottom). The time increases to the left. 


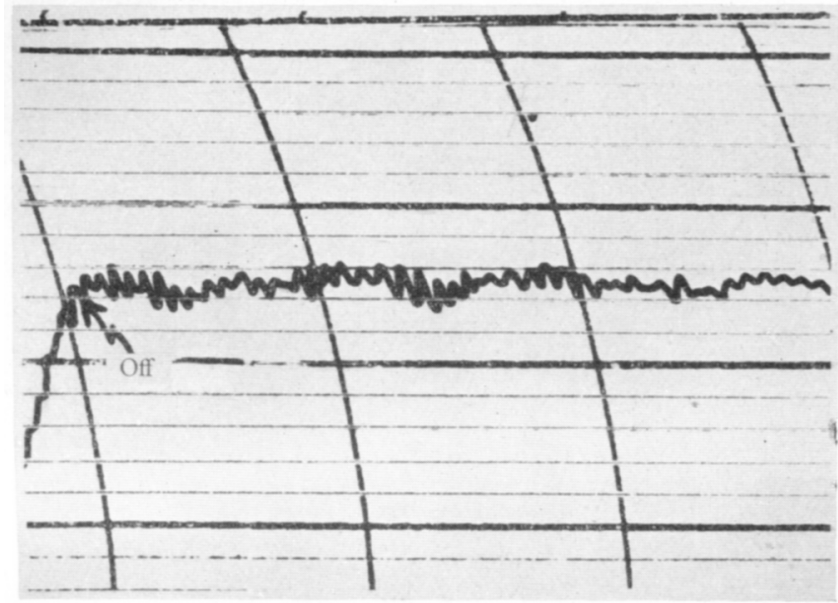

Plate III. Enlargement of the bottom trace shown in Pl. II. 


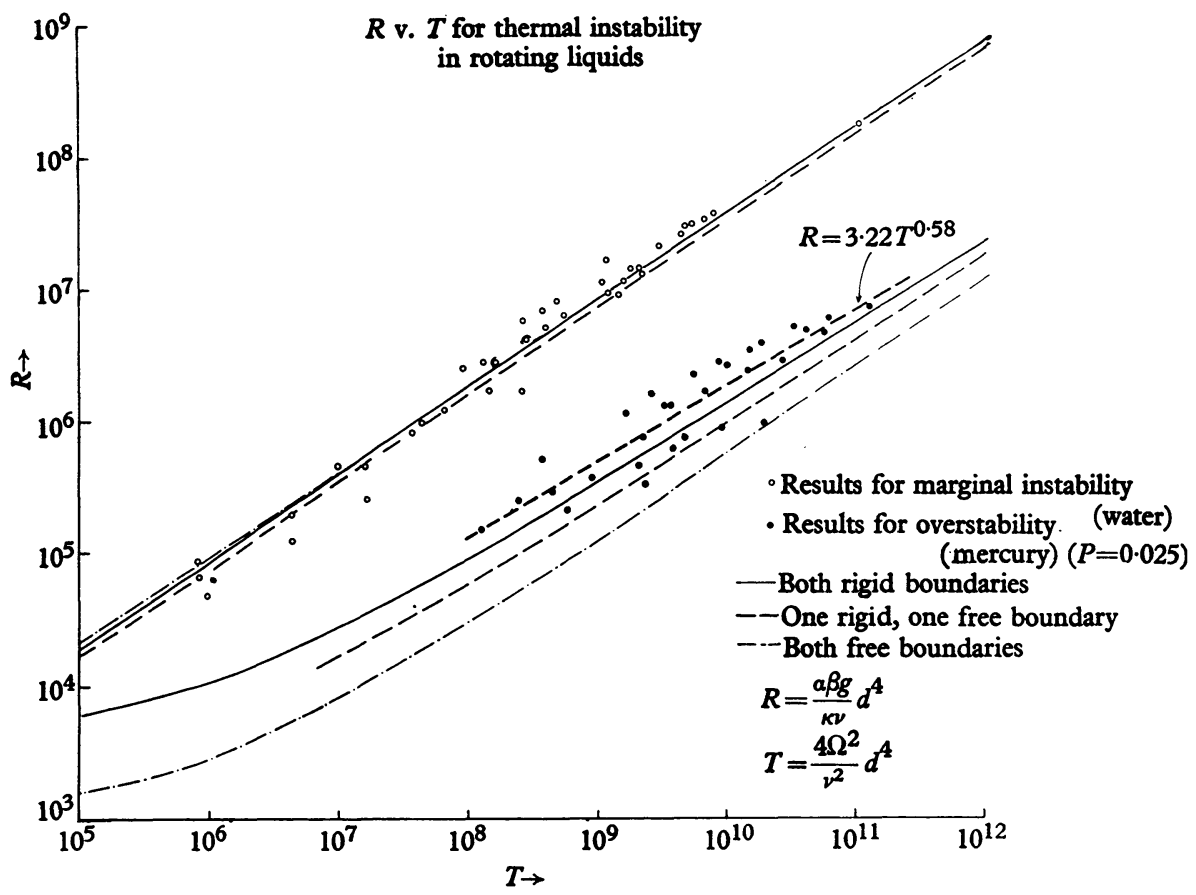

Fig. 2. Comparison of the predicted and observed Rayleigh numbers for rotating liquids as a function of the Taylor number T. (From:D. Fultz and Y. Nakagawa. [2].)

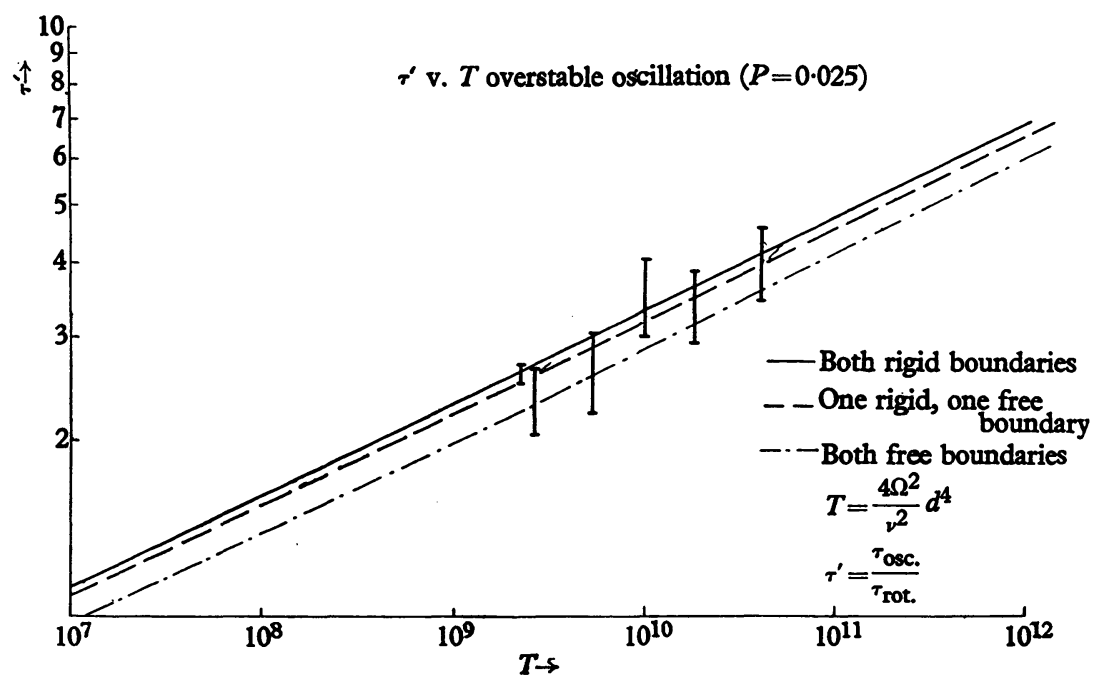

Fig. 3. Comparison of the predicted and observed periods of overstable oscillation for rotating layer of mercury. (From D. Fultz and Y. Nakagawa. [2].) 
predictions of $R_{c}$ and the periods of the overstable oscillations have been confirmed.

The last study of the series summarized in Table $\mathrm{I}$-the case where there is both a magnetic field and an angular velocity-is by far the most difficult. The theoretical treatment [9] involves the solution of a double eigenvalue problem of the twelfth order: for assigned $Q$ and $T$ both the critical Rayleigh number and the period of oscillation must be determined as eigenvalues. The experiment requires one to rotate the apparatus in a magnetic field which may reach 8000 gauss. Extreme precautions must be taken to eliminate inductive effects if $\beta$ is to be measured with the required accuracy (a few microvolts) by means of the thermopile.

\section{Table I}

\begin{tabular}{|c|c|c|c|}
\hline \multicolumn{2}{|c|}{ Experiment no. } & Convective case & Overstable case \\
\hline I & $\begin{array}{l}H=0 \\
\Omega=0\end{array}$ & $\begin{array}{l}R_{c} \text { is constant } \\
\text { Experimentally confirmed }\end{array}$ & Cannot occur \\
\hline II & $\begin{array}{l}H=0 \\
\Omega \neq 0\end{array}$ & $\begin{array}{l}R_{c} \propto T^{2} \text { as } T \rightarrow \infty \\
\text { Experimentally confirmed for water }\end{array}$ & $\begin{array}{l}R_{c}^{*} \propto T^{2} \text { as } T \rightarrow \infty \\
\text { Exists for } \nu \ll \kappa \\
\text { Experimentally confirmed for } \\
\text { mercury }\end{array}$ \\
\hline III & $\begin{array}{l}H \neq 0 \\
\Omega=0\end{array}$ & $\begin{array}{l}R_{\mathrm{c}} \propto Q \text { as } Q \rightarrow \infty \\
\text { Experimentally confirmed for } \\
\text { mercury }\end{array}$ & $\begin{array}{l}R_{c}^{*} \propto Q \text { as } Q \rightarrow \infty \\
\text { No experiment is possible, as } \eta \text { must } \\
\text { be less than } \kappa \text { for overstability to } \\
\text { occur. Occurs under astrophysical } \\
\text { conditions }\end{array}$ \\
\hline IV & $\begin{array}{l}H \neq 0 \\
\Omega \neq 0\end{array}$ & $\begin{array}{l}R_{c} \propto Q \text { as } Q \rightarrow \infty \\
\quad \text { for fixed } T\end{array}$ & $\begin{array}{l}R_{c}^{*} \propto Q \text { as } Q \rightarrow \infty \\
\text { for fixed } T \text {. Experiment in progress } \dagger\end{array}$ \\
\hline
\end{tabular}

It is feasible to compute the critical Rayleigh number only if both bounding surfaces are free. The results of the calculation are presented in Fig. 4; notice that the magnetic field (in a certain range of $Q$, for fixed $T$ ) may exert a destabilizing influence on the fluid. The wave numbers of the cells which appear at marginal stability are plotted in Fig. 5. As one increases the magnetic field (for fixed $T$ ) the cell size changes discontinuously by a factor which may be as great as Io. This change occurs at the same place where the slope of $R_{c}$ versus $Q$ changes abruptly in Fig. 4 .

As indicated in the table, the experiments relating to this problem have just begun in the new hydromagnetics laboratory. The experiment now in progress is designed to cover the overstable region in Fig. 4 and the abrupt transition to convection which should occur as the magnetic field is increased.

$\dagger$ Note added in proof: These experiments have now been completed by $Y$. Nakagawa and are reported in Proc. Roy. Soc. A, 240, 108, 1957; Proc. Roy. Soc. A, 242, 81, 1957; and Rev. Sci. Instrum. 21, 603, 1957. 


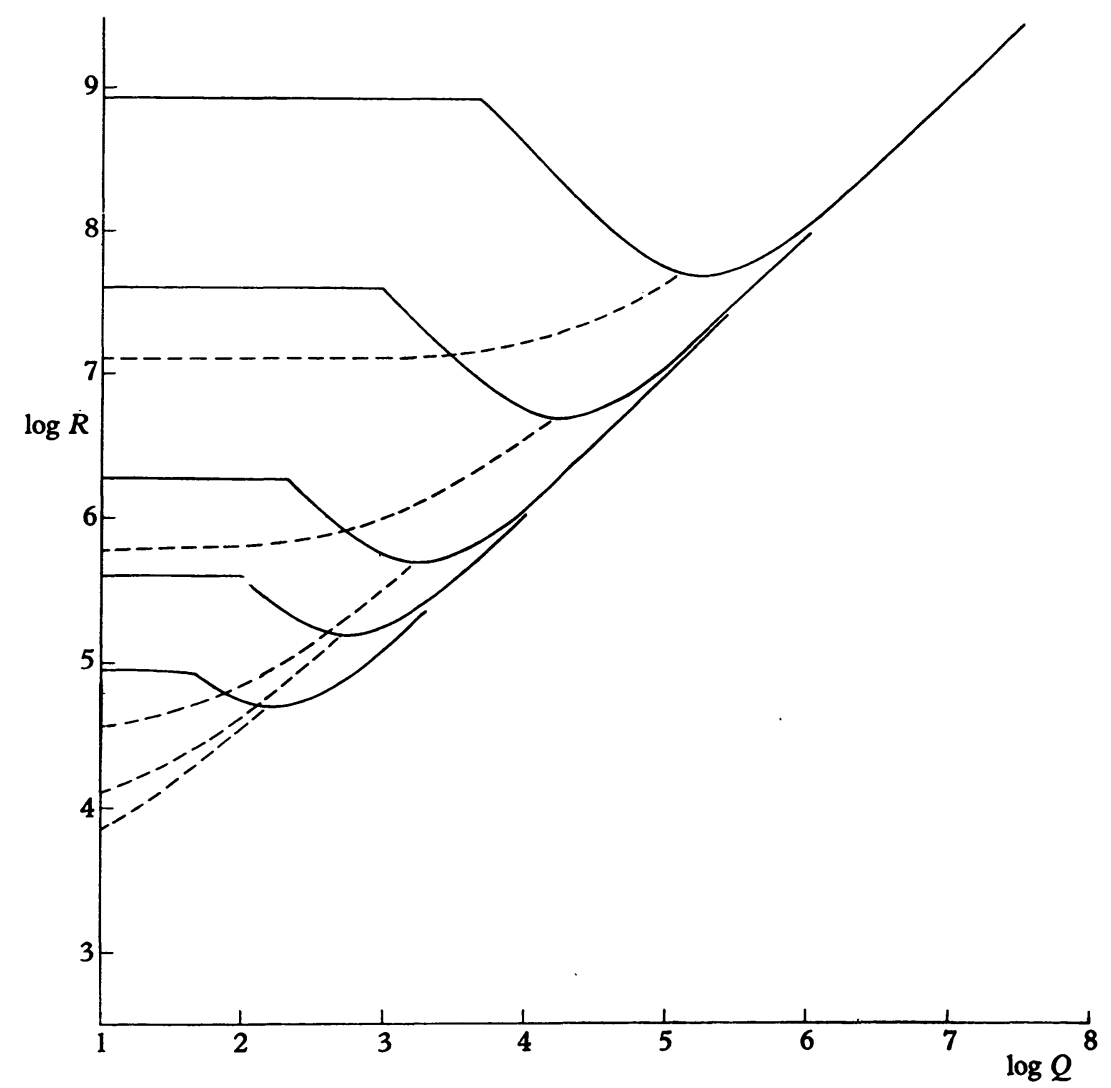

Fig. 4. Predicted critical Rayleigh number $R$ as a function of magnetic number $Q$ and Taylor number $T$. The Taylor numbers corresponding to each pair of curves are, from the top down, $10^{10}, 10^{8}, 10^{6}, 10^{5}, 10^{4}$. The dashed curves correspond to overstability, the full curves to convection. (From S. Chandrasekhar [9].)

\section{REFERENGES}

[1] Fultz, D., Nakagawa, Y. and Frenzen, P. Phys. Rev. 94, 147 I, I954.

[2] Fultz, D. and Nakagawa, Y. Proc. Roy. Soc. A, 23I, 2 I I, 1955.

[3] Nakagawa, Y. and Frenzen, P. Tellus, 7, I, I955.

[4] Nakagawa, Y. Nature, Lond. 175, 4I 7, 1955.

[5] Chandrasekhar, S. Phil. Mag. (7), 43, 501, 1952.

[6] Chandrasekhar, S. Proc. Roy. Soc. A, 217, 306, 1933.

[7] Chandrasekhar, S. Phil. Mag. (7), 45, 1177 , 1954.

[8] Chandrasekhar, S. Proc. Roy. Soc. A, 225, 173 , 1954.

[9] Chandrasekhar, S. Proc. Roy. Soc. A, 237, 476, 1956.

[10] Chandrasekhar, S. and Elbert, D. Proc. Roy. Soc. A, 231, 198, 1955.

\section{Discussion}

Biermann: When I discussed the subject of the cooling of sunspots by magnetic inhibition of convection, in agreement with Chandrasekhar, the results of this 
work, in a general way, seemed to support the line of reasoning underlying the theoretical conceptions. Are there any more recent results which might throw a light on this problem?

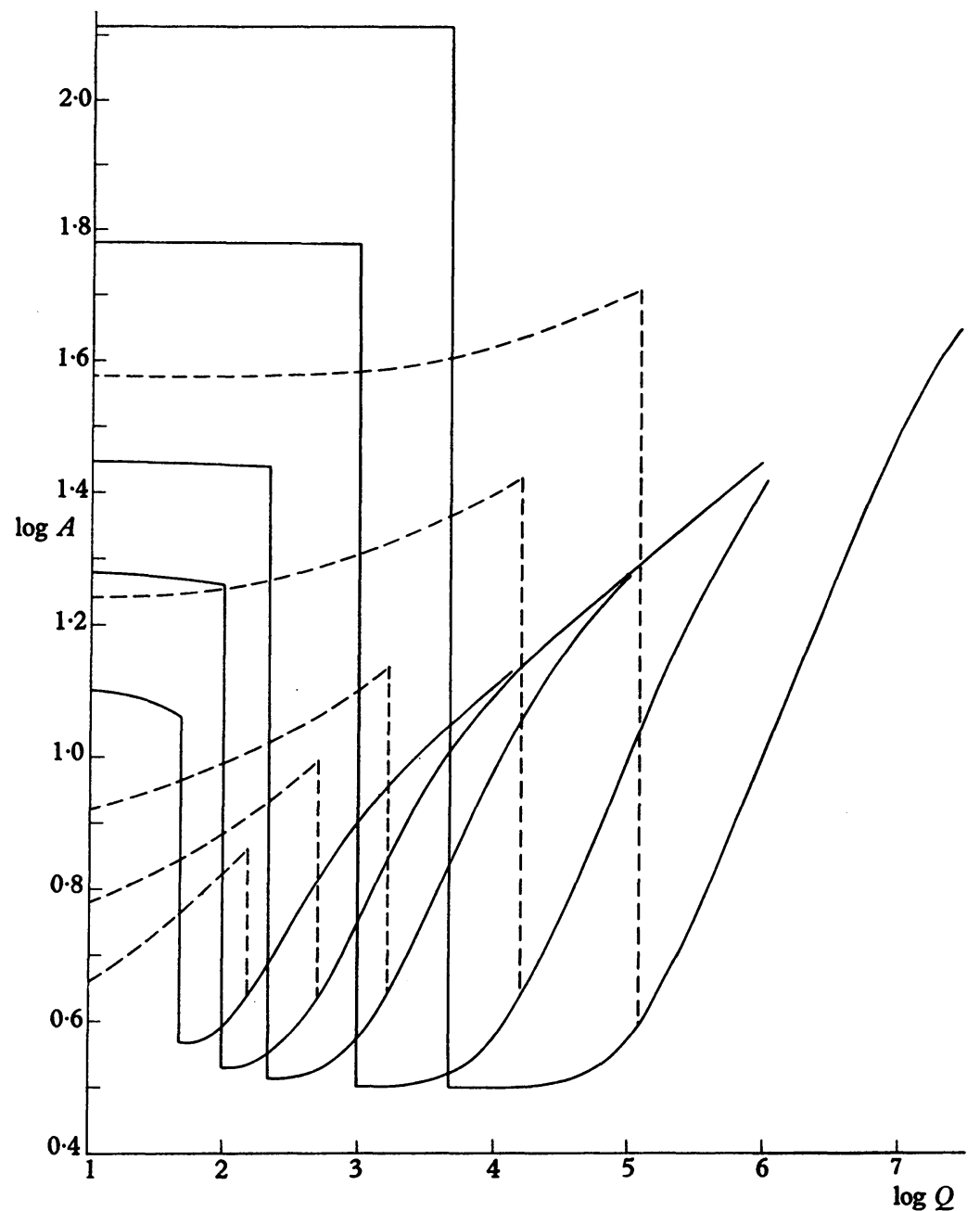

Fig. 5. Horizontal wave number at marginal stability as a function of $Q$ and $T$. The Taylor numbers are the same as those in Fig. 4. The dashed curves correspond to overstability, the full curves to convection. (From S. Chandrasekhar [9].)

Prendergast: I can only answer that the experiment has been extended over a much wider range of values than in earlier investigations by Nakagawa, and that it agrees with theory. The only case which has been examined is that without the influence of the Coriolis force. An experiment is being planned where a magnetic field and a rotation are acting simultaneously. This is, of course, the most interesting case. 
Lehnert: I should like to point out that convection on laboratory scale corresponds to aperiodic motions (as I have called them in my paper), whereas convective motions in astrophysics mainly consist of periodic modes. The former type of convection is critically suppressed by an external magnetic field, but not necessarily the latter. Consequently, the types of convection which can be realized in the laboratory are quite different from those to be considered in astrophysical problems, such as in sunspots. This has also been pointed out by Chandrasekhar.

Schatzman: Recently Rösch at Pic du Midi observed granulation inside sunspots. I want to ask Professor Biermann about his opinion of these observations.

Biermann: I talked with Rösch about his observations recently. It seemed then that more complete details must become known, e.g. the time scale of the spot granulae, before conclusions regarding the postulated magnetic inhibition of convection in the spots can be drawn.

Gold: Also one ought to mention the case of the penumbra. One would like to find a reason for a sudden change in the type of convection before its complete inhibition in order to account for the sharp distinction in the appearance of granulation, penumbra and umbra.

Lehnert: One might feel tempted to identify the regions of granulation, penumbra and umbra with states of turbulent convection, regular cellular convection and thermal conduction, respectively. The boundaries should then be given by the critical magnetic field strengths for the suppression of turbulent convection and of regular convection (Tellus, 9, I02, 1957). However, it is not at all certain that the onsets of regular convection and of turbulent convection are critical in the astrophysical situation.

Thompson: Are any attempts being made to observe cell shapes in a magnetic field?

Prendergast: No, there is no room for a mirror between the pole faces.

Thompson: Are there any experiments planned in which the magnetic field and the temperature gradient are not parallel; for in such a case the cell shape should be considerably changed?

Prendergast: An experiment is planned with vertical temperature gradient and a horizontal field.

Lehnert: A simple experiment with an oblique magnetic field has recently been performed in Stockholm (Tellus, 9, I01, 1957).

Hide: As Dr Prendergast has explained, when hydromagnetic forces or Coriolis forces act separately, the critical Rayleigh number, $R_{c}$, increases monotonically with the parameters $Q$ or $T$, as the case may be. Thus, these agencies, acting separately, tend to inhibit instability. However, when rotation and a magnetic field act simultaneously there are certain values of $T$ and $Q$ for which $R_{c}$ decreases with $T$ and $Q$. Is there any simple physical interpretation of this extremely interesting result that it is possible to choose values for the magnetic field and the rate of rotation such that any increase in them will encourage instability?

Prendergast: This point is clarified in a recent paper by S. Chandrasekhar, Dedalus, 86, 323, I957. 
Lehnert: I think that Dr Hide's question has some connexion with an earlier derived result (Quart. Appl. Math. 12, 335, 1955). Consider a plane hydromagnetic mode in an external magnetic field. We restrict the discussion to the aperiodic case, where the dissipation is too strong (or the field too weak) for eigen-oscillations to exist. Then, an increase in magnetic field strength causes an increase in the induced currents and in the dissipation. If a rotation is introduced the Coriolis force may be shown to counteract this effect; the increase of the dissipation caused by the magnetic field is reduced.

A possible physical explanation of this is that the Coriolis force provides a contribution to the effective 'tension' of the magnetic field lines, which may be regarded as elastic 'strings'. Thus, for a given momentum balance between the forces acting on a fluid element, the electrodynamical force can be reduced, part of its role being taken over by the Coriolis force. A reduction of the electrodynamic force gives a corresponding reduction in induced currents and in Joule heat dissipation. If this interpretation is right one could perhaps also state that, for a given value of $Q$, an increase of $T$ would destabilize the motion in some cases.

Lowes: Does overstability occur over the whole vessel simultaneously or at random in one or more convection cells? By using a thermopile you are presumably averaging the temperature fluctuations. Might you therefore miss some cases?

Prendergast: You certainly get a kind of averaging by using a thermopile. The cell dimension is, let us say, of the order of a centimeter and the thermopile causes an average over several cells. One gets an average of the fluctuations in temperature in cells. The order of fluctuation is $0.01 \% \mathrm{~cm}$ and the amplitude in an individual cell is probably considerably higher than that. From one cell to the next, under experimental realizable conditions and certainly not over the whole vessel, there need not be a whole region where the cells move like that. They move in one direction at one time and in another at another time. 